Int. J. Contemp. Math. Sciences, Vol. 9, 2014, no. 1, 1 - 10

HIKARI Ltd, www.m-hikari.com

http://dx.doi.org/10.12988/ijcms.2014.310116

\title{
11-Dissection and Modulo 11 Congruences Properties for Partition Generating Function
}

\author{
Goksal Bilgici \\ Kastamonu University, Education Faculty \\ Department of the Computer Education and Instructional Technology \\ 37100, Kastamonu, Turkey \\ gbilgici@kastamonu.edu.tr
}

Ali Bulent Ekin

Ankara University, Faculty of Science

Department of Mathematics

06100, Tandogan, Ankara, Turkey

ekin@science.ankara.edu.tr

Copyright (C) 2014 Goksal Bilgici and Ali Bulent Ekin. This is an open access article distributed under the Creative Commons Attribution License, which permits unrestricted use, distribution, and reproduction in any medium, provided the original work is properly cited.

\begin{abstract}
In a recent paper, we give 13-dissection and some congruences for modulo 13 for the partition generating function $\prod\left(1-q^{r}\right)^{-1}$ by using a method of Kolberg. In this paper, by following similar course, we develop an algoritmic approach and give 11-dissection for the partition generating function $\prod\left(1-q^{r}\right)^{-1}$. Then we re-obtain the congruences given by Atkin and Swinnerton-Dyer.
\end{abstract}

Mathematics Subject Classification: 11P83

Keywords: Partition, Dissections, $q$ - Equivalance 


\section{Introduction}

A partition of a positive integer $n$ is a non-increasing sequence of positive integers whose sum is $n$. The number of partitions of $n$ is denoted $p(n)$ and $p(0)$ is assumed as 1 . Euler gave the following generating function for the series $\{p(n)\}_{n=0}^{\infty}$

$$
\sum_{n=0}^{\infty} p(n) q^{n}=\prod_{r=1}^{\infty} \frac{1}{1-q^{r}} .
$$

Throughout this paper, $m>1$ always denotes a positive integer prime to 6 , and the variables $y$ and $q$ are always related to $y=q^{m}(|q|<1)$. We define

$$
\mathrm{F}:=\sum_{n=0}^{\infty} p(n) q^{n} .
$$

For $k=1,2, \ldots, m-1$, we define $k$-th component of $\mathrm{F}$ as follows:

$$
\mathrm{F}^{(k, m)}:=q^{k} \sum_{n=0}^{\infty} p(m n+k) y^{n},
$$

then we have a dissection for partition generating function, namely

$$
\mathrm{F}=\sum_{n=0}^{\infty} p(n) q^{n}=\sum_{k=0}^{m-1} \mathrm{~F}^{(k, m)} .
$$

Kolberg gave 5-dissection and 7-dissection for F. A simpler form of 7dissection for $\mathrm{F}$ was given by Ekin in his doctoral thesis [3]. In a recent paper [2], the authors obtained 13-dissection for $\mathrm{F}$ and some congruences for the components $\mathrm{F}^{(k, 13)}$ where $0 \leq k \leq 12$. In this paper, by following the same course in [2], we obtain 11-dissection for F. The 11-dissection for F may appear for the first time. After obtaining the components, using a $q$-equivalence given in [2], we obtain the congruences for the components $\mathrm{F}^{(k, 11)}(\bmod 11)$ given by Atkin and Swinnerton-Dyer in [1].

We prefer the following notation:

$$
\begin{aligned}
P(a) & =\left(y^{a} ; y^{m}\right)_{\infty}\left(y^{m-a} ; y^{m}\right)_{\infty} \\
P(0) & =\left(y^{m}, y^{m}\right)_{\infty}
\end{aligned}
$$

where

$$
(z ; q)_{\infty}=\prod_{r=1}^{\infty}\left(1-z q^{r-1}\right)
$$

and $a$ is not a multiple of $m . P(a)$ satisfies

$$
P(m-a)=P(a), \quad P(-a)=P(m+a)=-y^{-a} P(a) .
$$

For $m=11$, Atkin and Swinnerton-Dyer gave 
Theorem 1.1 For $m=11$, we have

$$
\begin{aligned}
\mathrm{F}^{(0,11)} & \equiv \frac{P(0)}{P(1)} \quad(\bmod 11) \\
\mathrm{F}^{(1,11)} & \equiv q \frac{P(0) P(5)}{P(2) P(3)} \quad(\bmod 11) \\
\mathrm{F}^{(2,11)} & \equiv 2 q^{2} \frac{P(0) P(3)}{P(1) P(4)} \quad(\bmod 11) \\
\mathrm{F}^{(3,11)} & \equiv 3 q^{3} \frac{P(0) P(2)}{P(1) P(3)} \quad(\bmod 11) \\
\mathrm{F}^{(4,11)} & \equiv 5 q^{4} \frac{P(0)}{P(2)} \quad(\bmod 11) \\
\mathrm{F}^{(5,11)} & \equiv 7 q^{5} \frac{P(0) P(4)}{P(2) P(5)} \quad(\bmod 11) \\
\mathrm{F}^{(6,11)} & \equiv 0 \quad(\bmod 11), \\
\mathrm{F}^{(7,11)} & \equiv 4 q^{7} \frac{P(0)}{P(3)} \quad(\bmod 11) \\
\mathrm{F}^{(8,11)} & \equiv 6 y q^{8} \frac{P(0) P(1)}{P(4) P(5)} \quad(\bmod 11) \\
\mathrm{F}^{(9,11)} & \equiv 8 q^{9} \frac{P(0)}{P(4)} \quad(\bmod 11) \\
\mathrm{F}^{(10,11)} & \equiv 9 q^{10} \frac{P(0)}{P(5)} \quad(\bmod 11)
\end{aligned}
$$

They use the following congruence to calculate Theorem 1.1:

$$
\sum_{k=0}^{10} \mathrm{~F}^{(k, 11)} q^{k}=(q ; q)_{\infty}^{-1} \equiv\left\{\prod\left(1-q^{r}\right)^{3}\right\}^{3} \prod\left(1-q^{r}\right) / \prod\left(1-y^{r}\right) \quad(\bmod 11)
$$

We calculate 11-dissection for the partition generating function at first. Then, we obtain the Theorem 1.1 by using the components with an algorithmic approach.

\section{Preliminaries}

Kolberg defines, for $s=0,1, \ldots, m-1$

$$
g_{s}:=\sum_{\frac{1}{2} n(3 n+1) \equiv s}(-1)^{n} q^{\frac{1}{2} n(3 n+1)}
$$


and

$$
h_{s}:=\sum_{\frac{1}{2} n(n+1) \equiv s(\bmod m),}(-1)^{n}(2 n+1) q^{\frac{1}{2} n(n+1)} .
$$

These definitions give

$$
\prod_{r=1}^{\infty}\left(1-q^{r}\right)=\sum_{s=0}^{m-1} g_{s} \text { and } \prod_{r=1}^{\infty}\left(1-q^{r}\right)^{3}=\sum_{s=0}^{m-1} h_{s} .
$$

By these equations, we conclude the following relation

$$
\left(g_{0}+g_{1}+\cdots+g_{m-1}\right)^{3}=h_{0}+h_{1}+\cdots+h_{m-1} .
$$

We have the following lemma from [4].

\section{Lemma 2.1}

$$
g_{s}=\left\{\begin{aligned}
0, & \text { if } 24 s+1 \text { is a quad. non-residue mod } m \\
(-1)^{\left[\frac{1}{6}(m+1)\right]} q^{\frac{1}{24}\left(m^{2}-1\right)} P(0), & \text { if } 24 s+1 \equiv 0 \quad(\bmod m)
\end{aligned}\right.
$$

and

$$
h_{s}=\left\{\begin{aligned}
0, & \text { if } 8 s+1 \text { is a quad. non-res. mod } m \\
(-1)^{\left[\frac{1}{2}(m-1)\right]} m q^{\frac{1}{8}\left(m^{2}-1\right)} P^{3}(0), & \text { if } 8 s+1 \equiv 0 \quad(\bmod m) .
\end{aligned}\right.
$$

Using the following lemma which is given by the authors in [2], we can determine the $g_{s}$ in terms of $P(a)$.

Lemma 2.2 Let $24 s+1$ is a quadratic residue $\bmod m$ and $m=6 \lambda+\mu$ where $\lambda$ is a positive integer and $\mu= \pm 1$. Then we have

$$
g_{s}=(-1)^{c+\lambda} q^{\frac{1}{2}\left(3 c^{2}-m c+3 \lambda^{2}+\mu \lambda\right)} \frac{P(2 c)}{P(c)}
$$

where $c$ is a solution of the congruence $x^{2} \equiv(4 s-\mu \lambda) / 6 \quad(\bmod m)$.

Kolberg also gives

Lemma 2.3 For $s=0,1, \ldots, m-1$

$$
\mathrm{F}^{(s, m)}=(-1)^{(m-1) s} \frac{P(0)}{(y ; y)_{\infty}^{m+1}} D_{s}
$$

where $D_{s}$ is the following determinant;

$$
\left|\begin{array}{cccc}
g_{-s} & g_{-s+1} & \cdots & g_{-s+m-2} \\
g_{-s-1} & g_{-s} & \cdots & g_{-s+m-3} \\
\cdots & \cdots & \cdots & \cdots \\
g_{-s-m+2} & g_{-s-m+3} & \cdots & g_{-s}
\end{array}\right|
$$

we put $g_{r}=g_{s}$ when $r \equiv s \quad(\bmod m)$ in $(7)$. 
We define

$$
A_{s}:=g_{k}^{1-m} D_{s}
$$

where $m$ is prime and $24 k+1 \equiv 0 \quad(\bmod m)$. So we have

$$
\mathrm{F}^{(s, m)}=q^{\frac{1}{24}\left(m^{3}-m^{2}-m+1\right)} \frac{P^{m}(0)}{(y ; y)_{\infty}^{m+1}} A_{s}
$$

For the denominator of (8), we have

$$
(y ; y)_{\infty}=\prod_{r=1}^{\infty}\left(1-y^{r}\right)=P(0) P(1) P(2) \cdots P((m-1) / 2) .
$$

We use the following lemma which is given by the authors in [2] to obtain the congruence properties of components.

Lemma 2.4 If $m \in \mathrm{Z}^{+}$is a prime, then

$$
\left[\prod_{r=1}^{\infty}\left(1-q^{r}\right)^{3}\right]^{m} \equiv P^{m+2}(0) P^{m+3}(1) P^{m+3}(2) \cdots P^{m+3}((m-1) / 2) \quad(\bmod m) \text {. }
$$

For the left-hand side on Eq.(10), we need the following lemma which is Lemma 3 in [1]:

Lemma 2.5 We have

$$
\prod_{r=1}^{\infty}\left(1-q^{r}\right)^{3} \equiv P(0) \sum_{c=0}^{(m-3) / 2}(-1)^{c}(2 c+1) q^{\frac{1}{2} c(c+1)} P\left(\frac{m-1}{2}-c\right) \quad(\bmod m) .
$$

\section{Components and Congruences for $m=11$}

In this section we give the components $\mathrm{F}^{(k, 11)}$ and find the congruences given by Atkin and Swinnerton-Dyer. For $m=11$, from (3) and (4) we have

$$
g_{3}=g_{6}=g_{8}=g_{9}=g_{10}=0, \quad g_{5}=q^{5} P(0)
$$

and

$$
h_{2}=h_{5}=h_{7}=h_{8}=h_{9}=0, \quad h_{4}=-11 q^{15} P^{3}(0) .
$$

We set

$$
\alpha:=g_{0} g_{5}^{-1}, \quad \beta:=g_{1} g_{5}^{-1}, \quad \gamma:=g_{2} g_{5}^{-1}, \quad \theta:=g_{4} g_{5}^{-1}, \quad \delta:=g_{7} g_{5}^{-1} .
$$


From (2) we find

$$
\begin{aligned}
3\left(2 g_{2} g_{4} g_{7}+2 g_{1} g_{5} g_{7}+g_{0} g_{1}^{2}+g_{4}^{2} g_{5}+g_{0}^{2} g_{2}\right) & =h_{2}=0, \\
3\left(2 g_{0} g_{1} g_{4}+2 g_{4} g_{5} g_{7}+g_{2} g_{7}^{2}+g_{1} g_{2}^{2}+g_{0}^{2} g_{5}\right) & =h_{5}=0, \\
3\left(2 g_{0} g_{2} g_{5}+2 g_{1} g_{2} g_{4}+g_{4} g_{7}^{2}+g_{0}^{2} g_{7}+g_{1}^{2} g_{5}\right) & =h_{7}=0, \\
3\left(2 g_{0} g_{1} g_{7}+2 g_{1} g_{2} g_{5}+g_{2}^{2} g_{4}+g_{5} g_{7}^{2}+g_{0} g_{4}^{2}\right) & =h_{8}=0, \\
3\left(2 g_{0} g_{4} g_{5}+2 g_{0} g_{2} g_{7}+g_{2}^{2} g_{5}+g_{1}^{2} g_{7}+g_{1} g_{4}^{2}\right) & =h_{9}=0, \\
3\left(g_{1} g_{7}^{2}+g_{0}^{2} g_{4}+g_{0} g_{2}^{2}+g_{4}^{2} g_{7}+g_{1}^{2} g_{2}\right)+g_{5}^{3} & =h_{4}=-11 g_{5}^{3} .
\end{aligned}
$$

By the help of (12), these equations become, respectively

$$
\begin{gathered}
2 \gamma \theta \delta+\alpha \beta^{2}+\alpha^{2} \gamma+\theta^{2}+2 \beta \delta=0, \\
2 \alpha \beta \theta+\gamma \delta^{2}+\beta \gamma^{2}+\alpha^{2}+2 \theta \delta=0, \\
2 \beta \gamma \theta+\theta \delta^{2}+\alpha^{2} \delta+\beta^{2}+2 \alpha \gamma=0, \\
2 \alpha \beta \delta+\alpha \theta^{2}+\gamma^{2} \theta+\delta^{2}+2 \beta \gamma=0 \\
2 \alpha \gamma \delta+\beta \theta^{2}+\beta^{2} \delta+\gamma^{2}+2 \alpha \theta=0, \\
\alpha^{2} \theta+\beta^{2} \gamma+\theta^{2} \delta+\gamma^{2} \alpha+\delta^{2} \beta=-4 .
\end{gathered}
$$

Now we put

$$
x_{1}:=\alpha^{2} \theta, \quad x_{2}:=\beta^{2} \gamma, \quad x_{3}:=\theta^{2} \delta, \quad x_{4}:=\gamma^{2} \alpha, \quad x_{5}:=\delta^{2} \beta .
$$

Thus (18) becomes

$$
x_{1}+x_{2}+x_{3}+x_{4}+x_{5}=-4 .
$$

After multiplying $A_{s}(s=0,1, . ., 10)$ by $\alpha, \alpha^{-1}, \beta^{-1}, \gamma^{-1}, \delta, \theta^{-1}, 1, \theta, \delta^{-1}, \gamma$ and $\beta$ respectively, $A_{s}$ can be written in terms of $x_{i}$.

From (3) and (5), we get

$$
\begin{aligned}
& g_{0}=\frac{P(0) P(4)}{P(2)}, g_{1}=-q \frac{P(0) P(2)}{P(1)}, g_{2}=-q^{2} \frac{P(0) P(5)}{P(3)}, \\
& g_{4}=-q^{4} y \frac{P(0) P(1)}{P(5)}, g_{5}=q^{5} P(0), g_{7}=q^{7} \frac{P(0) P(3)}{P(4)} .
\end{aligned}
$$

These equations give

$$
\alpha \beta \gamma \theta \delta=-1
$$

and

$$
x_{1} x_{2} x_{3} x_{4} x_{5}=-1 .
$$

Lemma 3.1 We have

$$
\begin{aligned}
& x_{1} x_{2}=x_{4}+1, \\
& x_{2} x_{3}=x_{5}+1, \\
& x_{3} x_{4}=x_{1}+1, \\
& x_{4} x_{5}=x_{2}+1, \\
& x_{5} x_{1}=x_{3}+1 .
\end{aligned}
$$


Proof. We define $A:=x_{3} x_{4} x_{5}, B:=x_{1} x_{2} x_{5}, C:=x_{1} x_{4} x_{5}, D:=x_{1} x_{2} x_{3}$ and $E:=x_{2} x_{3} x_{4}$. Multiplying equations (13), (14), (15), (16) and (17) by $\delta, \theta, \gamma$, $\beta$ and $\alpha$ respectively give us

$$
\begin{aligned}
& 2 A+B+C=x_{3}+2 x_{5}, \\
& 2 D+A+E=x_{1}+2 x_{3}, \\
& 2 E+A+C=x_{2}+2 x_{4}, \\
& 2 B+D+E=x_{5}+2 x_{2}, \\
& 2 C+D+B=x_{4}+2 x_{1} .
\end{aligned}
$$

The solution of this equations system is

$$
\begin{aligned}
& A=x_{3} x_{4} x_{5}=\frac{1}{4}\left(-x_{1}-x_{2}-x_{4}+3 x_{3}+3 x_{5}\right), \\
& B=x_{1} x_{2} x_{5}=\frac{1}{4}\left(-x_{1}+3 x_{2}-x_{4}-x_{3}+3 x_{5}\right), \\
& C=x_{1} x_{4} x_{5}=\frac{1}{4}\left(3 x_{1}-x_{2}+3 x_{4}-x_{3}-x_{5}\right), \\
& D=x_{1} x_{2} x_{3}=\frac{1}{4}\left(3 x_{1}-x_{2}-x_{4}+3 x_{3}-x_{5}\right), \\
& E=x_{2} x_{3} x_{4}=\frac{1}{4}\left(-x_{1}+3 x_{2}+3 x_{4}-x_{3}-x_{5}\right) .
\end{aligned}
$$

Using equation (20), we get

$$
\begin{aligned}
& x_{3} x_{4} x_{5}=x_{3}+x_{5}+1, \\
& x_{1} x_{2} x_{5}=x_{2}+x_{5}+1, \\
& x_{1} x_{4} x_{5}=x_{1}+x_{4}+1, \\
& x_{1} x_{2} x_{3}=x_{1}+x_{3}+1, \\
& x_{2} x_{3} x_{4}=x_{2}+x_{4}+1 .
\end{aligned}
$$

Multiplying both sides of (27) by $x_{1} x_{2}$ and using (21), we find

$$
-1=x_{1} x_{2} x_{3}+x_{1} x_{2} x_{5}+x_{1} x_{2} .
$$

Substituting for (28), (30) and (20) into the equation (32), we obtain

$$
x_{1} x_{2}=x_{4}+1
$$

and we find the others similarly.

Algorithm 1. Let $U$ be a linear combination of $x_{1}^{i_{1}} x_{2}^{i_{2}} x_{3}^{i_{3}} x_{4}^{i_{4}} x_{5}^{i_{5}}$ where each $i_{r}$ is a non-negative integer. 
1. Substitute for the equations (22)-(26) into $U$. This step turns all terms into the form $x_{i}^{a}$ or $x_{i}^{a} x_{j}^{b}$ where $(i, j)=(1,4),(2,5),(4,2),(3,1),(5,3)$.

2. If $b>1$ then substitute for $x_{j}^{2}$, if $a>1$ and $\mathrm{b}=0$ substitute for $x_{i}^{2}$ into $U$. This step writes $U$ as sums of $x_{i}^{a} x_{j}$ and $x_{i}$. To evaluate this step we use the following equations which can be easily found by (20):

$$
\begin{aligned}
& x_{1}^{2}=-x_{1} x_{4}-x_{3} x_{1}-4 x_{1}-x_{4}-x_{3}-2, \\
& x_{2}^{2}=-x_{4} x_{2}-x_{2} x_{5}-4 x_{2}-x_{4}-x_{5}-2, \\
& x_{3}^{2}=-x_{3} x_{1}-x_{5} x_{3}-4 x_{3}-x_{5}-x_{1}-2, \\
& x_{4}^{2}=-x_{1} x_{4}-x_{4} x_{2}-4 x_{4}-x_{1}-x_{2}-2, \\
& x_{5}^{2}=-x_{5} x_{3}-x_{2} x_{5}-4 x_{5}-x_{2}-x_{3}-2 .
\end{aligned}
$$

By using Algorithm 1, we get the $A_{s}$ in simple forms:

$$
\begin{aligned}
\alpha A_{0}= & -x_{4}^{4} x_{2}+31 x_{1}^{3} x_{4}+42 x_{3}^{3} x_{1}-42 x_{4}^{3} x_{2}-5 x_{5}^{3} x_{3}+172 x_{1}^{2} x_{4}+277 x_{3}^{2} x_{1} \\
& +10 x_{2}^{2} x_{5}-296 x_{4}^{2} x_{2}-64 x_{5}^{2} x_{3}+508 x_{1} x_{4}+724 x_{3} x_{1}-332 x_{4} x_{2} \\
& +199 x_{2} x_{5}-429 x_{5} x_{3}-362 x_{1}-1132 x_{2}-1464 x_{3}-116 x_{4} \\
& -1147 x_{5}-3970, \\
\alpha^{-1} A_{1}= & 7 x_{2}^{4} x_{5}-8 x_{4}^{3} x_{2}+3 x_{1}^{3} x_{4}-22 x_{5}^{3} x_{3}+140 x_{2}^{3} x_{5}+2 x_{3}^{3} x_{1}+28 x_{4}^{2} x_{2} \\
& +85 x_{1}^{2} x_{4}-180 x_{5}^{2} x_{3}+433 x_{2}^{2} x_{5}+74 x_{3}^{2} x_{1}-113 x_{4} x_{2}+234 x_{4} x_{1} \\
& +1004 x_{5} x_{2}-524 x_{5} x_{3}+176 x_{1} x_{3}+562 x_{4}+705 x_{5}+578 x_{2}+345 x_{1} \\
& -158 x_{3}+1224 \\
A_{6}= & 11\left[-x_{1}^{3} x_{4}-x_{2}^{3} x_{5}-x_{4}^{3} x_{2}-x_{3}^{3} x_{1}-x_{5}^{3} x_{3}-14 x_{1}^{2} x_{4}-14 x_{2}^{2} x_{5}\right. \\
& -14 x_{4}^{2} x_{2}-14 x_{3}^{2} x_{1}-14 x_{5}^{2} x_{3}-29 x_{1} x_{4}-29 x_{2} x_{5}-29 x_{2} x_{4} \\
& \left.-29 x_{1} x_{3}-29 x_{3} x_{5}+106\right] .
\end{aligned}
$$

The last equation proves the famous congruence $p(11 n+6) \equiv 0 \quad(\bmod 11)$ of Ramanujan.

If we observe the indices of the terms in $A_{s}$, having $\alpha A_{0}$ and $\alpha^{-1} A_{1}$ is enough to obtain other components via the permutation (12345). This permutation gives the following relations

$$
\begin{gathered}
\alpha A_{0} \rightarrow \beta A_{10} \rightarrow \theta A_{7} \rightarrow \gamma A_{9} \rightarrow \delta A_{4} \rightarrow \alpha A_{0} \\
\alpha^{-1} A_{1} \rightarrow \beta^{-1} A_{2} \rightarrow \theta^{-1} A_{5} \rightarrow \gamma^{-1} A_{3} \rightarrow \delta^{-1} A_{8} \rightarrow \alpha^{-1} A_{1} \\
A_{6} \rightarrow A_{6} .
\end{gathered}
$$

Using these relations, that is, changing indices in convenient order and making the same operations, we get the other components. For $m=11$, with the help of Eq.(10) and Lemma 2.5, we have

$$
\begin{gathered}
P^{11}(5)+8 y P^{11}(4)+5 y^{3} P^{11}(3)+4 y^{6} P^{11}(2)+9 y^{10} P^{11}(1) \equiv \\
P^{2}(0) P^{14}(1) P^{14}(2) P^{14}(3) P^{14}(4) P^{14}(5) \quad(\bmod 11) .
\end{gathered}
$$


For abbreviation, we define

$$
\left(a_{1}, a_{2}, \cdots, a_{(m+1) / 2}\right):=y^{a_{1}} P^{a_{2}}(1) P^{a_{3}}(2) P^{a_{(m+1) / 2}}((m-1) / 2) .
$$

We write (43) in terms of $x_{i}$ in 20 different ways by dividing (43) by $(a, b, c, d, e, f)$ where $1 \leq b, c, d, e, f \leq 5$ and $b+c+d+e+f=11$. Five of them are useful for us and the remaining 15 of them are linearly independent on these five. These can be found dividing (43) by $(3,1,2,3,2,3)$, $(4,2,2,3,3,1),(4,2,3,1,3,2),(4,3,1,2,2,3)$ and $(5,3,3,2,1,2)$ :

$$
\begin{array}{r}
7 x_{2}^{6} x_{3}^{5} x_{1} x_{4}+2 x_{3}^{6} x_{4}^{4} x_{2}+3 x_{1}^{5} x_{2}^{4} x_{3}+10 x_{4}^{5} x_{5}^{3} x_{3}+6 x_{5}^{4} x_{1}^{3} \\
\equiv(y ; y)_{\infty}^{2}(-3,13,12,11,12,11) \quad(\bmod 11), \\
9 x_{3}^{6} x_{4}^{5} x_{2} x_{5}+x_{4}^{6} x_{5}^{4} x_{3}+7 x_{2}^{5} x_{3}^{4} x_{4}+5 x_{5}^{5} x_{1}^{3} x_{4}+3 x_{1}^{4} x_{2}^{3} \\
\equiv(y ; y)_{\infty}^{2}(-4,12,12,11,11,13) \quad(\bmod 11), \\
x_{4}^{6} x_{5}^{5} x_{1} x_{3}+5 x_{5}^{6} x_{1}^{4} x_{4}+2 x_{3}^{5} x_{4}^{4} x_{5}+3 x_{1}^{5} x_{2}^{3} x_{5}+4 x_{2}^{4} x_{3}^{3} \\
\equiv(y ; y)_{\infty}^{2}(-4,12,11,13,11,12) \quad(\bmod 11), \\
8 x_{1}^{6} x_{2}^{5} x_{3} x_{5}+7 x_{2}^{6} x_{3}^{4} x_{1}+5 x_{5}^{5} x_{1}^{4} x_{2}+10 x_{4}^{4} x_{5}^{3}+2 x_{3}^{5} x_{4}^{3} x_{2} \\
\equiv(y ; y)_{\infty}^{2}(-4,11,13,12,12,11) \quad(\bmod 11), \\
5 x_{5}^{6} x_{1}^{5} x_{2} x_{4}+3 x_{1}^{6} x_{2}^{4} x_{5}+10 x_{4}^{5} x_{5}^{4} x_{1}+4 x_{2}^{5} x_{3}^{3} x_{1}+9 x_{3}^{4} x_{4}^{3} \\
\equiv(y ; y)_{\infty}^{2}(-5,11,11,12,13,12) \quad(\bmod 11) .
\end{array}
$$

By using Algorithm 1 to the left hand side of these congruences, we obtain

$$
\begin{aligned}
& 3 x_{1}^{4} x_{4}+5 x_{1}^{3} x_{4}+6 x_{1} x_{3}^{3}+4 x_{2}^{3} x_{5}+6 x_{3} x_{5}^{3}+8 x_{1}^{2} x_{4}+x_{1} x_{3}^{2}+5 x_{2}^{2} x_{5} \\
& +3 x_{2} x_{4}^{2}+5 x_{3} x_{5}^{2}+6 x_{1} x_{4}+5 x_{1} x_{3}+8 x_{2} x_{4}+6 x_{3} x_{5}+6 x_{1}+8 x_{2}+7 x_{4} \\
& +7 x_{3}+2 x_{5}+4 \quad \equiv(y ; y)_{\infty}^{2}(-3,13,12,11,12,11) \quad(\bmod 11) \\
& 7 x_{2}^{4} x_{5}+3 x_{1}^{3} x_{4}+2 x_{1} x_{3}^{3}+8 x_{2}^{3} x_{5}+3 x_{2} x_{4}^{3}+8 x_{1}^{2} x_{4}+8 x_{1} x_{3}^{2}+4 x_{2}^{2} x_{5} \\
& +6 x_{2} x_{4}^{2}+7 x_{3} x_{5}^{2}+3 x_{1} x_{4}+8 x_{2} x_{4}+3 x_{2} x_{5}+4 x_{3} x_{5}+x_{1}+3 x_{2}+9 x_{4} \\
& +4 x_{3}+9 x_{5}+2 \quad \equiv(y ; y)_{\infty}^{2}(-4,12,12,11,11,13) \quad(\bmod 11) \\
& 2 x_{1} x_{3}^{4}+7 x_{1} x_{3}^{3}+4 x_{2}^{3} x_{5}+10 x_{2} x_{4}^{3}+4 x_{3} x_{5}^{3}+2 x_{1}^{2} x_{4}+9 x_{1} x_{3}^{2}+7 x_{2}^{2} x_{5} \\
& +7 x_{2} x_{4}^{2}+8 x_{3} x_{5}^{2}+9 x_{1} x_{4}+4 x_{1} x_{3}+4 x_{2} x_{5}+7 x_{3} x_{5}+x_{1}+5 x_{2}+9 x_{4} \\
& +4 x_{3}+x_{5}+10 \quad \equiv(y ; y)_{\infty}^{2}(-4,12,11,13,11,12) \quad(\bmod 11) \\
& 5 x_{3} x_{5}^{4}+3 x_{1}^{3} x_{4}+10 x_{2}^{3} x_{5}+10 x_{2} x_{4}^{3}+x_{3} x_{5}^{3}+x_{1}^{2} x_{4}+5 x_{1} x_{3}^{2}+9 x_{2}^{2} x_{5}+x_{2} x_{4}^{2} \\
& +6 x_{3} x_{5}^{2}+6 x_{1} x_{3}+10 x_{2} x_{4}+x_{2} x_{5}+10 x_{3} x_{5}+6 x_{1}+8 x_{2}+7 x_{4}+8 x_{3} \\
& +10 x_{5}+3 \quad \equiv(y ; y)_{\infty}^{2}(-4,11,13,12,12,11) \quad(\bmod 11) \\
& 10 x_{2} x_{4}^{4}+9 x_{1}^{3} x_{4}+9 x_{1} x_{3}^{3}+2 x_{2} x_{4}^{3}+6 x_{3} x_{5}^{3}+7 x_{1}^{2} x_{4}+2 x_{1} x_{3}^{2}+10 x_{2}^{2} x_{5} \\
& +x_{2} x_{4}^{2}+2 x_{3} x_{5}^{2}+2 x_{1} x_{4}+9 x_{1} x_{3}+9 x_{2} x_{4}+x_{2} x_{5}+5 x_{1}+5 x_{2}+9 x_{4} \\
& +3 x_{3}+x_{5}+6 \quad \equiv(y ; y)_{\infty}^{2}(-5,11,11,12,13,12) \quad(\bmod 11)
\end{aligned}
$$

Now we can obtain the congruences given by Atkin and Swinnerton-Dyer. From (39), we have

$$
\alpha A_{0} \equiv 10 x_{2} x_{4}^{4}+9 x_{1}^{3} x_{4}+9 x_{1} x_{3}^{3}+2 x_{2} x_{4}^{3}+6 x_{3} x_{5}^{3}+7 x_{1}^{2} x_{4}+2 x_{1} x_{3}^{2}+10 x_{2}^{2} x_{5}
$$




$$
\begin{aligned}
& +x_{2} x_{4}^{2}+2 x_{3} x_{5}^{2}+2 x_{1} x_{4}+9 x_{1} x_{3}+9 x_{2} x_{4}+x_{2} x_{5}+x_{1}+x_{2}+5 x_{4}+10 x_{3} \\
& +8 x_{5}+1 \quad(\bmod 11)
\end{aligned}
$$

From (48), we find

$$
A_{0} \equiv \alpha^{-1}(y ; y)_{\infty}^{2}(-5,11,11,12,13,12) \quad(\bmod 11)
$$

Then the equations (8) and (49) give us

$$
\mathrm{F}^{(0,11)} \equiv \frac{P(0)}{P(1)} \quad(\bmod 11) .
$$

The other congruences in Theorem 1.1 can be obtained similarly.

\section{References}

[1] A.O.L. Atkin and H.P.F. Swinnerton-Dyer, Some Properties of Partitions, Proc. London Math. Soc., 3 (4) (1954), 84-106. http://dx.doi.org/10.1112/plms/s3-4.1.84

[2] G. Bilgici and A.B. Ekin, Some Congruences for Modulus 13 Related to Partition Generating Function, The Ramanujan Journal, (to be appear). http://dx.doi.org/10.1007/s11139-013-9537-4

[3] A.B. Ekin, The Rank and the Crank in the Theory of Partition, D.Phil Thesis, University of Sussex, 1993.

[4] O. Kolberg, Some Identities Involving the Partition Function, Math. Scand., 5 (1957), 77-92.

Received: November 1, 2013 